\title{
Annette Leibing und Silke Schicktanz (Hrsg) (2020) Preventing Dementia? - Critical Perspectives on a New Paradigm of Preparing for Old Age
}

\author{
Berghahn Books, New York/Oxford, 268 Seiten, $145 €$, ISBN \\ 978-1-78920-909-9
}

\section{Eike Buhr (D)}

Angenommen: 20. Januar 2022 / Online publiziert: 26. Januar 2022

(C) Der/die Autor(en) 2022

Laut eines Lancet-Reports aus dem Jahr 2017 kann einer von drei Fällen von Demenz verhindert werden, sofern 12 Risikofaktoren beachtet werden (Livingstone et al. 2017). Dabei ist nicht nur die hohe Anzahl der verhinderbaren Fälle oder die genaue Benennung der Risikofaktoren erstaunlich, sondern auch die Art der Risikofaktoren: Faktoren der persönlichen Lebensführung. Demenz erscheint somit nicht mehr als Schicksal, sondern als Ergebnis von beeinflussbaren „Lifestyle Faktoren“.

Vor diesem Hintergrund diagnostizieren die Herausgeberinnen des Bandes Preventing Dementia? - Critical Perspectives on a New Paradigm of Preparing for Old Age, Annette Leibing und Silke Schicktanz, eine Veränderung des wissenschaftlichen und auch medialen Diskurses zur Demenzprävention. Diese als sicher erscheinende, aber dennoch kontrovers diskutierte Sichtweise auf Demenzprävention, bei gleichzeitiger epistemischer, ätiologischer, diagnostischer und therapeutischer Unsicherheit, bezeichnen sie als ,new dementia“. Der interdisziplinäre Band versammelt zehn Beiträge internationaler Autoren und Autorinnen, die sich mit dieser neuen Betrachtungsweise der Prävention demenzieller Erkrankungen aus unterschiedlichen Perspektiven auseinandersetzen, und ist in drei Abschnitte geteilt.

Der erste Abschnitt zu den sozialen und diskursiven Praktiken der Demenzprävention beginnt mit dem Beitrag von Lara Keuck, die aus historischer Perspektive darlegt, inwiefern Diagnose und klinisches Krankheitsbild auch die Wahrnehmung von Alzheimer-Demenz in der Öffentlichkeit prägen. Sie schlägt vor, transparent mit diagnostischen und klinischen blinden Flecken umzugehen. Anette Leibing geht in ihrem Beitrag dem Ursprung des ,,preventive turns“ in der Demenzforschung auf den Grund. Dabei identifiziert sie als Gründe neben epistemischen Grundlagen der Ätiologie demenzieller Erkrankungen ebenfalls ökonomische Interessen. Zugleich zeigt

Eike Buhr $(\bowtie)$

Abteilung Ethik in der Medizin, Carl-von-Ossietzky Universität Oldenburg, Oldenburg, Deutschland

E-Mail: eike.buhr@uni-oldenburg.de 
sie, dass sich die „,new dementia“ auch in Schuldzuweisungen ausdrückt und strukturelle Kritik am Gesundheitssystem sekundär bleibt. Silke Schicktanz untersucht in ihrem Beitrag den ,,preventive turn“ anhand des deutschsprachigen wissenschaftlichen und medialen Diskurses. Sie identifiziert nicht nur Unterschiede zwischen den Diskursen, sondern bescheinigt dem medialen Diskurs auch, die ,epistemische Vagheit“" der Wirksamkeit präventiver Maßnahmen auszublenden. Hiervon ausgehend plädiert sie für eine veränderte ethische Auseinandersetzung, die von voreiligen Verantwortungszuschreibungen absieht. Matthias Leanza setzt sich in seinem Beitrag mit der Kritik an der Darstellung von Demenz als vermeidbarer Alterserscheinung mit der Betonung von Eigenverantwortung und individuellen Schuldzuweisungen auseinander. Überzeugend legt er dar, dass die Schaffung des ,,preventive self“ nicht bloß das Produkt individueller Verantwortungszuschreibung ist, sondern am Ende einer langen Kette gesellschaftlicher Prozesse und kollektiver Anstrengungen steht. Alessandro Blasimme erörtert in seinem Beitrag die epistemischen Grundlagen des neueren klinischen Diskurses über Demenzprävention. Statt des Verfolgens einer geriatrischen Logik, im Zuge derer der Verlust kognitiver Fähigkeiten entlang einer breiteren Linie des Verfalls altersbedingter körperlicher Funktion betrachtet wird, schlägt er einen ganzheitlichen Ansatz vor, der die Stärkung der Resilienz und Vitalkräfte der Person zum Ziel hat.

Der zweite Abschnitt setzt sich mit dem Verhältnis von Vorhersage, Früherkennung und der Prävention von Demenz auseinander. Tiago Moreira verfolgt in seinem Beitrag die Entwicklung von Mild Cognitive Impairment (MCI) als Hauptrisikofaktor für die Entwicklung von Alzheimer zu einer Kategorie, die lose mit verschiedenen Ätiologien verbunden ist. Auch Stephen Katz, Kevin R. Peters und Peri J. Ballantyne beschäftigen sich in ihrem Beitrag mit der Unklarheit und Instabilität von MCI und erörtern anhand von Interviews mit Expert:innen aus Medizin, Beobachtungen des pharmazeutischen Marktes sowie Fokusgruppen, warum MCI dennoch weiterhin als Diagnosekategorie verwendet wird.

Den Auftakt des dritten Abschnittes, der den konzeptuellen Vorannahmen und normativen Implikation der Prävention gewidmet ist, macht Kirsten Bell mit ihrem Beitrag. Sie zeigt am Beispiel der USA auf, vor welchem Hintergrund ,lifestyle changes" als Präventionsmaßnahmen auch für demenzielle Erkrankungen in Betracht gezogen werden. Mark Schweda und Larissa Pfaller kontextualisieren in ihrem Beitrag den Diskurs um Demenzprävention mit den Diskursen um „successful aging“ und ,active-aging“. In diesem Zusammenhang analysieren sie, unter welchen Bedingungen die Zuschreibung individueller Verantwortung für die eigene Gesundheit problematisch ist. Im abschließenden Beitrag untersucht Thomas Foth aus genealogischer Perspektive den Fokus auf Lifestylefaktoren als entscheidendes Merkmal der ,new dementia“. Er argumentiert, dass, entgegen der verbreiteten Annahme, die Betonung von Lifestylefaktoren und die damit einhergehende Responsibilisierung individuellen Gesundheitsverhaltens nicht mit einem Rückzug des Staates aus der Gesundheitsversorgung verbunden ist, sondern die Aufgaben des Staates sich in diesem Kontext im Sinne einer ,neoliberalen Govermentalität“ lediglich gewandelt haben.

Der Sammelband gibt einen guten Überblick über die mit der „,new dementia“ verbundenen Diskurse und analysiert überzeugend und kenntnisreich die Diskrepanz 
zwischen öffentlicher sowie zum Teil wissenschaftlicher Darstellung und faktischen Erfolgsaussichten der Demenzprävention durch Lifestylefaktoren. Die damit einhergehende individuelle Responsibilisierung wird schlüssig kontextualisiert und ethisch bewertet. Durch das Aufzeigen der Verschränkung der medizinischen Demenzprävention mit epistemischen, sozialen, historischen und kulturellen sowie ökonomischen Faktoren eröffnen die einzelnen Beiträge wichtige Impulse für die Auseinandersetzung mit der ,new dementia“, die weiterhin dringend erforderlich ist. Der Sammelband ist somit nicht nur für Fachleute medizinischer Praxis, sondern auch für die Medizinethik, -geschichte und -soziologie von großem Interesse.

Funding Open Access funding enabled and organized by Projekt DEAL.

Open Access Dieser Artikel wird unter der Creative Commons Namensnennung 4.0 International Lizenz veröffentlicht, welche die Nutzung, Vervielfältigung, Bearbeitung, Verbreitung und Wiedergabe in jeglichem Medium und Format erlaubt, sofern Sie den/die ursprünglichen Autor(en) und die Quelle ordnungsgemäß nennen, einen Link zur Creative Commons Lizenz beifügen und angeben, ob Änderungen vorgenommen wurden.

Die in diesem Artikel enthaltenen Bilder und sonstiges Drittmaterial unterliegen ebenfalls der genannten Creative Commons Lizenz, sofern sich aus der Abbildungslegende nichts anderes ergibt. Sofern das betreffende Material nicht unter der genannten Creative Commons Lizenz steht und die betreffende Handlung nicht nach gesetzlichen Vorschriften erlaubt ist, ist für die oben aufgeführten Weiterverwendungen des Materials die Einwilligung des jeweiligen Rechteinhabers einzuholen.

Weitere Details zur Lizenz entnehmen Sie bitte der Lizenzinformation auf http://creativecommons.org/ licenses/by/4.0/deed.de.

Interessenkonflikt E. Buhr gibt an, dass kein Interessenkonflikt besteht.

\section{Literatur}

Livingston G, Sommerlad A, Orgeta V, Costafreda SG, Huntley J, Ames D, Ballard C et al (2017) Dementia prevention, intervention, and care. Lancet 390(10113):2673-2734. https://doi.org/10.1016/S01406736(17)31363-6 